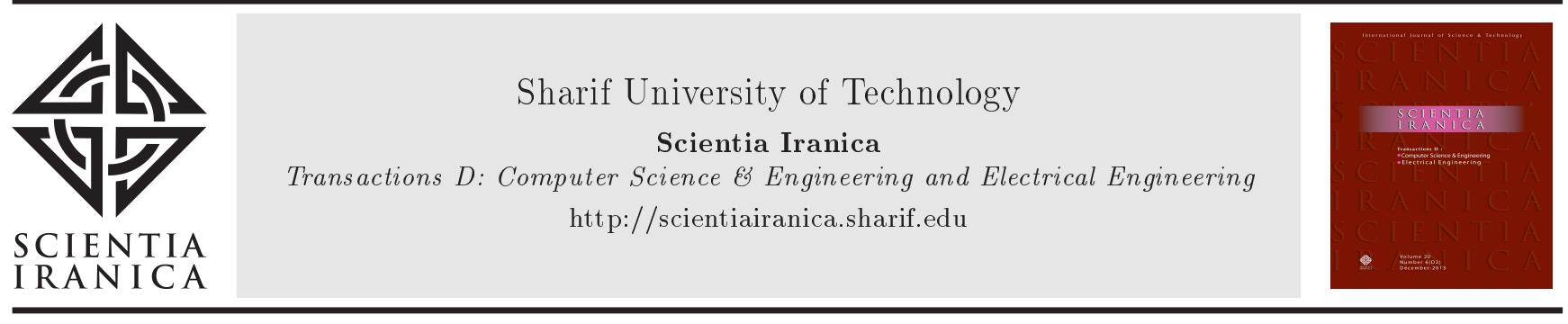

\title{
A new approach to the setting of directional overcurrent relays by incorporating cascading outages
}

\author{
H. Karimkhan Zand ${ }^{\mathrm{a}, \mathrm{b}}$, K. Mazlumi ${ }^{\mathrm{a}}$, and A. Bagheri ${ }^{\mathrm{a}, *}$ \\ a. Department of Electrical Engineering, Faculty of Engineering, University of Zanjan, Zanjan, Iran. \\ b. Zanjan Electricity Distribution Company (ZEDC), Zanjan, Iran.
}

Received 29 November 2019; received in revised form 30 April 2020; accepted 13 July 2020

\author{
KEYWORDS \\ Directional \\ overcurrent relays; \\ Distribution networks; \\ Uncertainty; \\ Cascading outages; \\ Multi-objective \\ optimization; \\ Genetic algorithm.
}

\begin{abstract}
Directional Overcurrent Relays (DOCRs) are essential protective devices in distribution networks which are usually set without considering any contingencies. However, the current challenge in power systems is the existence of uncertainty and its unfavorable consequences. It sometimes appears that some elements simultaneously fail which makes other parts overloaded to the extent that it leads to cascading outages. Therefore, DOCRs may malfunction, which ends in unwanted trips when there is no fault, or they may not operate in case the fault is located within their reach. In such cases, the coordination setting needs complex programming with many related non-linear inequality constraints. In this paper, a novel hybrid method is proposed based on multi-objective optimization including new objective functions using Genetic Algorithm (GA). Also, the cascading outages are considered in the presented method based on network data analysis. This approach is performed on distribution part of the IEEE 14-bus meshed system and a real industrial radial feeder named TOSEE, located in Iran. The simulations were implemented in MATLAB and PowerFactory-DIgSILENT software packages in different models and the results are evaluated.
\end{abstract}

(C) 2022 Sharif University of Technology. All rights reserved.

\section{Introduction}

The primary aim of using Directional Overcurrent Relays (DOCRs) in distribution networks is to detect the permanent faults and clear them through line sectionalizing using Circuit Breakers (CBs). Due to proper operation and low expenditure, DOCRs are the main protective devices in distribution networks. The decision variables of DOCRs include pickup current $(I p)$ and Time Multiplier Setting (TMS). In a microprocessor-based DOCRs, TMS changes continu-

*. Corresponding author.

E-mail address: amir_bagheri@znu.ac.ir (A. Bagheri) ously (due to the manufacturing advancement of the relaying devices), and this has a vital effect on the relays' operation time and Ip has discrete values that are proportional to Current Transformer Ratio (CTR).

The optimization methods with inherent advantages are employed to designate the valid values of the Protection Coordination Problem (PCP) [1,2]. Given that conventional methods have low convergence speed and low accuracy, they are no longer utilized [3].

Various optimization methods have been introduced to determine the decision variables of DOCRs. In [4], the decision variables were obtained by executing mathematical optimization processes in GAMS software. On the other hand, the $T M S$ values were attained in [5] by applying linear programming assuming identified $I p$ values. 
Recently, the optimization algorithms based on intelligence which are inspired by nature have been recommended for improving the efficacy of the method [6]. Consequently, both $I p$ and $T M S$ values were determined in $[7,8]$ using Genetic Algorithm (GA) by considering PCP as a non-linear programming. Alternatively, in $[9,10]$, PCP was formulated as a mixed nonlinear integral problem regarding the discrete values of the pickup current and the non-linear nature of the DOCRs' equation. Thus, this problem was solved using meta-heuristic optimization algorithms such as hybrid GA and Particle Swarm Optimization (PSO) in MATLAB software.

Besides, an enhanced protection coordination scheme was proposed in [11] utilizing Dual TimeCurrent Characteristics (DTCCs) for overcurrent relays which were followed by two inverse-time curves in series. These characteristics are fulfilled by numerical DOCRs incurring investment costs. Therefore, a techno-economic optimal replacement model is devised to attain the least-cost numerical DOCR deployment plan while eliminating miscoordination and minimizing the total operation time of the relays. The proposed model constitutes a Mixed Integer NonLinear Programming (MINLP) problem which is solved using sine-cosine algorithm. Furthermore, authors in [12] optimized different issues of overcurrent relays in microgrids. Two cases are taken for each relay: single variable (Time Dial Settings (TDS)) and two variables (TDS and pickup current setting $(I p)$ ). An objective function that minimizes the total operating time of all primary relays has been considered. A GA is employed for the optimization purpose which exhibits better performance than other conventional algorithms.

The conventional protection approaches are based on the fixed network topology [9]. However, the network topology may be altered following the maintenance or contingencies (including transient and permanent faults) or the operator's mistake. The contingencies such as line outage, Distributed Generation (DG) outage, and disconnection of sub-transmission network (islanding mode) increase the number of constraints that are likely to be violated, and this complicates the solution algorithm [13]. In [14,15], the decision variables were obtained for PCP in the sub-transmission network by considering all topologies derived from lines outage. Also, sometimes some power devices simultaneously fail and this leads to cascading outages, turning into a blackout. Although the likelihood of such an event is low, it may happen and result in substantial financial damage [16]. As an example, there were blackouts in Europe on November 4, 2006 and in India on July 30-31, 2012 [17,18]. Besides, it is estimated that annually more than 10 million people are incurred with financial damage due to the power grids uncertainty [19]. Therefore, it is appropriate to consider simultaneous contingencies in the DOCRs setting so that the network does not experience serious interruption.

Up to now, previous studies have not considered cascading outages through Multi-Objective Optimization Method (MOOM). The MOOM includes new Objective Functions (OFs) which are capable of solving $\mathrm{PCP}$ with the minimum violated constraints.

Moreover, the penetration of DGs into power systems is growing which affects the operation of distribution networks. Hence, by considering DG, the problem formulation should be extended. The employed DGs usually have synchronous generator types which have a greater impact on the network Short Circuit Level (SCL) compared to the inverter-based ones [20]. Synchronous-Based DGs (SBDGs) are more susceptible to power grid faults regarding their low inertia time constants. Researchers in [21] proposed communication-assisted dual-setting DOCRs as effective solutions for providing fast-response protection in order to meet transient stability constraints of SBDGs in microgrids.

In this paper, a novel hybrid method is introduced to protect and coordinate DOCRs by employing the MOOM, which involves new OFs. Moreover, all the cascading outages are investigated into different models and the decision variables are obtained for each one of them. The uncertainty within the power system is a challenging issue in the coordination problem that requires complex programming with many related nonlinear constraints. Therefore, one of the most critical aims is that the maximum number of these constraints should be satisfied. Unlike the current protection coordination methods, the present approach obtains decision variables by incorporating network data analysis and the GA with the purpose of optimizing the $O F s$ by seeking the best potential solution. The proposed approach is implemented on TOSEE radial feeder and the IEEE 14-bus system by incorporating DGs via PowerFactory-DIgSILENT and MATLAB software packages.

The outstanding features of this paper are outlined in the following:

- Solving the PCP based on MOOM;

- Considering the cascading outages;

- Defining new objective functions for PCP; and

- Applying the proposed method to a real distribution network besides the IEEE 14-bus system.

\section{Problem formulation}

\subsection{Relay's characteristic}

The DOCRs have numerous characteristics that have been investigated in the literature by employing stan- 
dard Inverse Definite Minimum Time (IDMT) overcurrent relay curves. Here, a non-linear one is recommended based on the IEC standard with a Standard Inverse (SI) tripping curve as in the following:

$$
t_{i}=\frac{0.14}{\left(\frac{I s c}{I p}\right)^{0.02}-1} \times T M S
$$

where $t_{i}$ is the $i$ th relay's operation time for a fault close to CBs and $I s c$ is the short-circuit current passing through the $i$ th relay.

\subsection{Problem constraints}

The protection-coordination problem is subject to some constraints which are described in the following.

\subsubsection{Coordination Time Interval (CTI)}

As shown in Figure 1, to have a time interval between the operation of the main relay $(\mathrm{Rm})$ and its backup relays $\left(\mathrm{Rb}^{1}, \mathrm{Rb}^{2}\right.$, and $\left.R \mathrm{Rb}^{n}\right)$, the CTI is considered. The backup relays function only in case of failure in the operation of the main relay. The CTI ensures the permanence of the protection coordination and the network's safety. The value of CTI is assumed 0.3 seconds in Eq. (2) by taking into account some factors such as the relay overshooting, CBs' interrupting time, and reliability of the network [22]. In Eq. (2), $t_{i}^{b}$ and $t_{i}^{m}$ are the main and backup relays' operation times, respectively. For each backup relay in the network, Eq. (3) must be satisfied. The relevant constraints can be expressed as follows:

$$
\begin{aligned}
& \Delta t m b=t_{i}^{b}-t_{i}^{m}-C T I, \\
& \Delta t m b \geq 0 .
\end{aligned}
$$

\subsubsection{The pickup current bounds}

The pickup current is limited to a particular range in Eq. (4) by taking into account the Current Transformer (CT) ratio $(0.5,0.6,0.8,1,1.5,2$, and 2.5) [13]. The fundamental concern in the protection of lines is that no line should be out of service due to the maloperation of DOCRs. Therefore, Ip must be larger

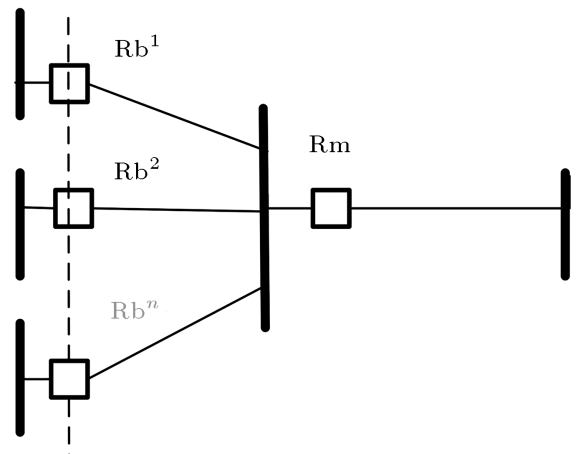

Figure 1. Main and backup relays. than the maximum load current in Relation (5) and the load flow ought to be executed by incorporating the peak load of the network. Moreover, Relation (6) ensures that DOCRs distinguish all the faults that occur in their reach point and detect the lowest shortcircuit current $\left(I s c_{\min }\right)$. However, in some particular conditions, the fault current is less than the maximum load current and Relation (6) leads to load interruption in case of no fault in the reach point of DOCRs. Thus, Relation (5) is preferred over Relation (6) as the load of power grid should not be sacrificed for the detection of this fault. This type of fault is named high-impedance fault. Therefore, the priority of the distribution networks is the loads' reliability and the reduction of interruption duration which can increase the consumers' satisfaction [23]. The bounds of Ip are defined as follows:

$$
\begin{aligned}
& I p_{\text {min }}<I p<I p_{\text {min }}, \\
& \text { Iload }_{\text {max }}<I p, \\
& I p>I s c_{\text {min }} .
\end{aligned}
$$

\subsection{3. $T M S$ bound}

The value of TMS is assumed to be continuous, and it is directly proportional to the relay's operation time based on the relay's characteristic. According to Relation (7), this variable is defined within lower and upper boundaries between 0.05 and 1 for all DOCRs:

$$
\text { TMSmin }<T M S<T M S \max .
$$

\subsection{Objective functions}

OFs are to satisfy all or most of the constraints corresponding to the protection and coordination and to ensure no miscoordination in the operation of the DOCRs. In this method, PCP is solved by taking into account the multi-objective optimization, which involves four new objective functions, $O F_{A}, O F_{B}$, $O F_{C}, O F_{D}$ according to Functions (8)-(11). It should be noted that these $O F s$ have not been used in the previous studies. The problem formulation aims to optimize all of them such that the best possible solutions are achieved simultaneously for each of $O F s$. Also, square brackets (i.e., [ ]) are used to denote the floor function, which rounds a real number down to the next integer (as in $[\pi]=3$, in $[-\pi]=-4$ ).

- $O F_{A}$. Miscoordination between the main relay and backup relays violates Relation $(3)(\Delta t m b<0)$, and $O F_{A}$ becomes large due to the high value assigned to $\alpha$. In this condition, the proposed algorithm will eliminate these chromosomes (decision variables) in the next generation until the best values are obtained. Moreover, this function tries to decrease the overall operation time (i.e., $\sum_{i=1}^{N R} t_{i}$ ). 
- $O F_{B}$. Similarly, an improper $T M S$ value (i.e., $T M S>1$ ) has a significant effect on PCP which is penalized by $O F_{B}$.

- $O F_{C}$. If the current passing through the main relay $\left(I p^{R m}\right)$ is remarkably higher than its backup relay's current $\left(I p^{R b}\right)$ (i.e., $I p^{R m} \geq 2 \times I p^{R b}$ ), the protection coordination is not reasonable due to the high value yield for $\Delta t m b(\Delta t m b>>0.3)$. Therefore, $O F_{C}$ is used for resolving this problem such that these chromosomes are not generated in the next iteration (generation).

- $O F_{D}$. Also, if the fault current is very close to $I p$ of the DOCRs (i.e., $I s c \leq I p \times 1.6$ ), it is not adopted for the protection coordination due to the higher operation time attained for DOCRs. Consequently, $O F_{D}$ is employed to solve such problems.

$$
\begin{aligned}
& O F_{A}=\alpha \times\left(\left|\left[\frac{\left|\Delta t_{m b}\right|}{1.1 \times \Delta t_{m b}}\right]\right|\right)+\sum_{i=1}^{N R} t_{i}, \\
& O F_{B}=\alpha \times([T M S i]), i=1, \ldots, N R, \\
& O F_{C}=\alpha \times\left(\left[\frac{I p^{R m}}{I p^{R b} \times 2}\right]\right), \\
& O F_{D}=\alpha \times\left(\left[\frac{I p \times 1.6}{I s c}\right]\right) .
\end{aligned}
$$

In Eqs. (8)-(11), $N R$ is the number of DOCRs. The parameter $\alpha$ is a weighting coefficient which is determined experimentally through trial and error. In this regard, a specific value of $\alpha$ may result in an increase in the relay's operation time, while it brings about a reduction in the number of miscoordination. Therefore, the considered $\alpha$ should choose either decreasing operation time of the relays or decreasing mis-coordination among them. Regarding this issue, the value of $\alpha$ has been considered equal to 500 in this paper. Besides, the reason why $\alpha$ is the same in all the objective functions is that all these four $O F s$ have the same weighting to have equal effects on the problem. It should be noted that we can leave the determination of $\alpha$ 's value to the optimization algorithm. In Subsection 4.5.1, this issue will be discussed and the coefficients will be optimized along with the objective function.

\section{The proposed approach for solving protection coordination}

The algorithm for determining decision variables of DOCRs in the distribution networks is formed as a non-linear programming. All the related constraints must be observed to acquire optimum values. The GA as a robust approach to optimization problems is employed to solve such a non-convex problem based on the generation of elite descendants. The decision variables are encoded within a collection of genes composing the chromosome. The GA generates a set of chromosomes which are known as a population. The initial population is randomly generated such that the maximum number of problem constraints is satisfied. All the generated chromosomes are chosen through selection, cross-over, and mutation operators for producing the next generation, and those having the most appropriate $O F s$ are preferred to be present in the next iteration. The full description of the GA was given in [24]. As seen in Figure 2, the flowchart of the suggested method is divided into three sections.

- First section. At first, the values of $I p$ are determined by incorporating the network peak load and CTR. Further, the fault current is calculated for all the main and backup relays according to the fault close to the CBs (i.e., network data analysis);

- Second section. Then, the TMS values encoded in the chromosomes of the GA are generated based on MOOM. In the case of a single-objective optimization problem, the solution aims to promote a performance index reflecting the quality of the solution by minimum or maximum Objective Function $(\mathrm{OF})$. However, sometimes, due to the nonlinear nature of the problem, we can consider several $O F s$ and optimize their values simultaneously for pursuing the best possible results. At each iteration in Section 2,OF $O F_{A}$ and $O F_{B}$ are evaluated, and among the $T M S$ values, those having the best values are selected for the next step;

- Third section. In the third section regarding the algorithm, based on the current settings obtained from the previous section, the new values of current and time settings are generated by the GA algorithm. In doing so, the obtained current settings fall in a proper range based on their lower and upper bounds, and the new values are produced by the GA, i.e., the $T M S$ and $I p$ values attained from Sections 1 and 2 are considered to be on a chromosome, and the final fitness values are obtained based on MOOM with all OFs $\left(O F_{A}, O F_{B}, O F_{C}, O F_{D}\right)$ by seeking the best potential solution.

The process continues in this manner until the required iterations are completed.

\section{Numerical studies}

In this part, the proposed approach to PCP is applied to two case studies in different models. The first case study is the distribution part of IEEE 14-bus system, while the second one is a real distribution network. Both case studies have DGs that participate in supplying loads of the system. The IEEE meshed system 


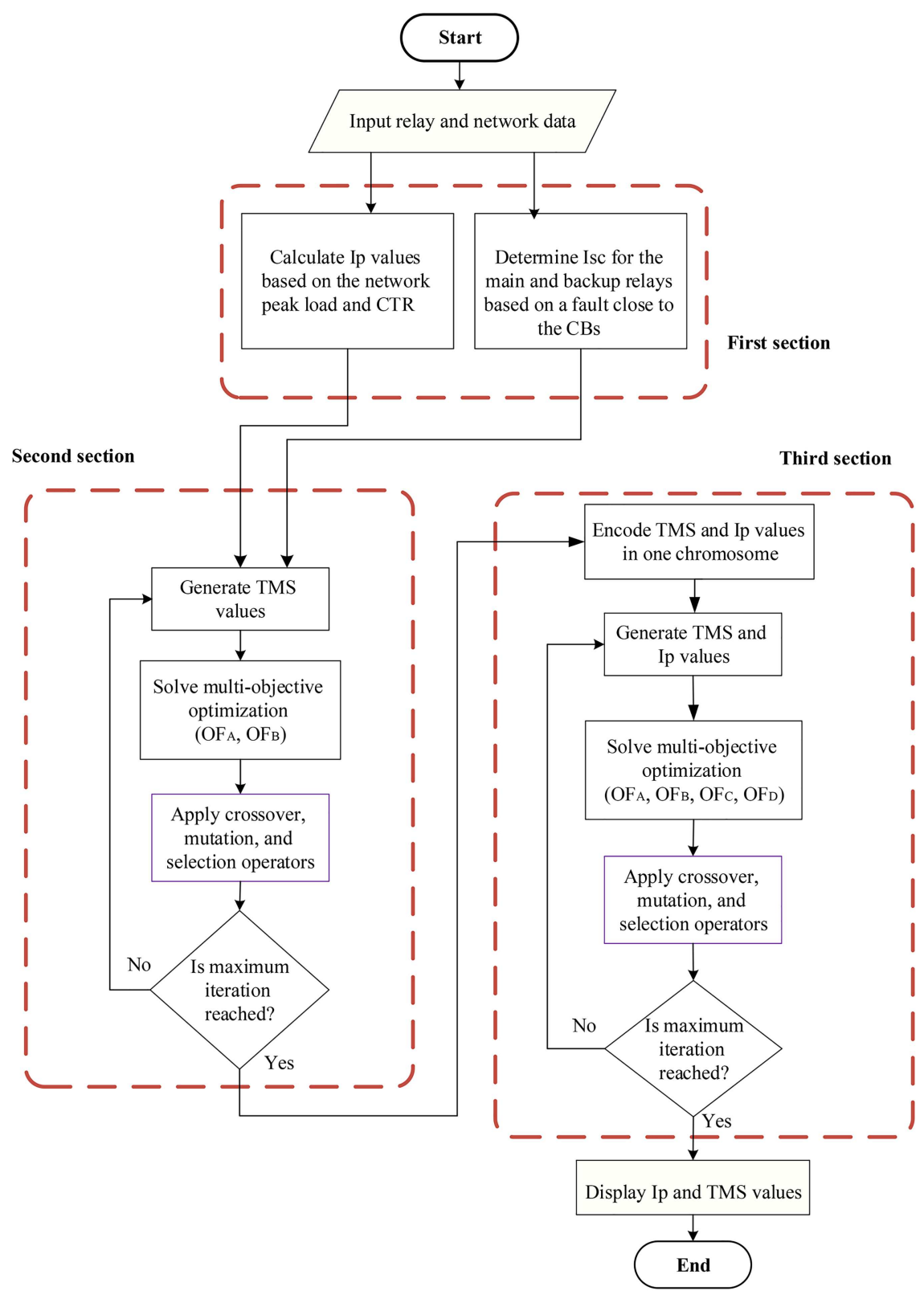

Figure 2. Flowchart of the proposed algorithm.

and TOSEE feeder have sixteen $(\mathrm{R} 1, \mathrm{R} 2, \ldots, \mathrm{R} 16)$ and four ( $\mathrm{Ra}, \mathrm{Rb}, \mathrm{Rc}, \mathrm{Rd}$ ) DOCRs, respectively, for all of which the $T M S$ and $I p$ values must be calculated. As mentioned earlier, the GA has been employed for the optimization purpose. The parameters of the employed GA in this paper are given in Table 1. It should be noted that these parameters have been tuned using trial and error through several executions of the GA to obtain the best results.

\subsection{The IEEE 14-bus system}

The first case study, shown in Figure 3, is the distribution part of IEEE 14-bus system as a microgrid. The under-study area has been distinguished by dashed lines within the whole system. The system
Table 1. Parameters of the employed genetic algorithm.

\begin{tabular}{cc}
\hline Parameter & Value/description \\
\hline Population size & 500 \\
Number of generations & 1000 \\
Elite counts & $5 \%$ \\
Crossover rate & $80 \%$ \\
Mutation type & Constraint dependent \\
Selection method & Stochastic uniform \\
\hline
\end{tabular}

data were given in [25]. This network is connected to a sub-transmission network via two $132 \mathrm{kV} / 33 \mathrm{kV}$ transformers having the capacity of 60 MVA. Three 10 MVA synchronous-generator-type DG units are also connected to this grid. Each DG unit is composed of 10 


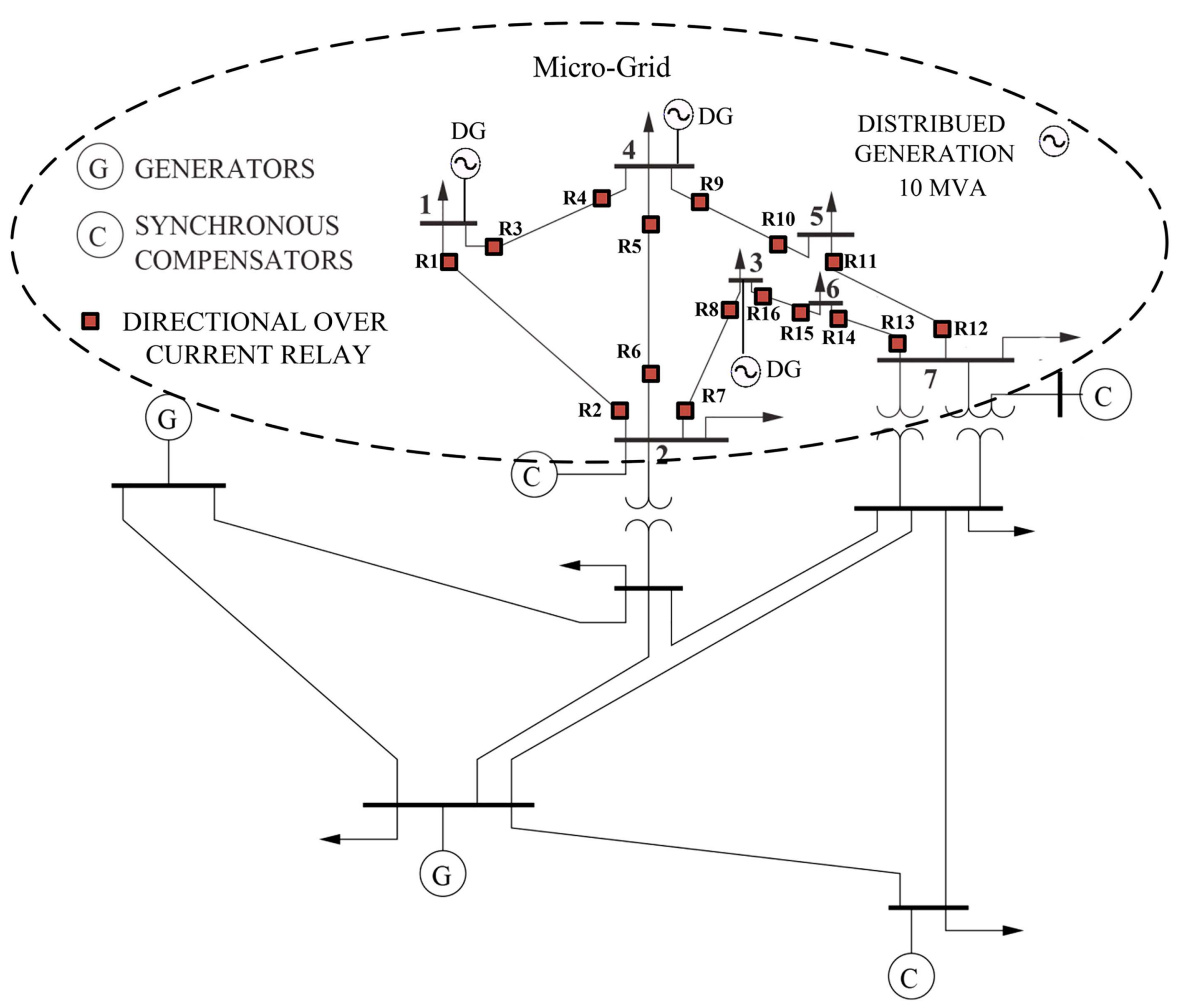

Figure 3. IEEE 14-bus network and its distribution part (micro-grid).

Table 2. Technical data of DG units connected to the microgrid.

\begin{tabular}{cccc}
\hline Parameter & Feature/value & Parameter & Feature/value \\
\hline Generator type & Gas-engine & Synchronous reactance, $X_{d}$ & $3.498 \mathrm{pu}$ \\
Rated capacity & $1 \mathrm{MVA}$ & Transient reactance, $X_{d}^{\prime}$ & $0.2935 \mathrm{pu}$ \\
Rated voltage & $0.4 \mathrm{kV}$ & Sub-transient reactance, $X_{d}^{\prime \prime}$ & $0.274 \mathrm{pu}$ \\
Rated current & $1.47 \mathrm{kA}$ & Zero-sequence reactance, $X_{0}$ & $0.0419 \mathrm{pu}$ \\
Frequency & $50 \mathrm{~Hz}$ & Negative-sequence reactance, $X_{2}$ & $0.1531 \mathrm{pu}$ \\
Nominal power factor & $0.93 \mathrm{lag}$ & Rotor type & Round-rotor \\
Earthling type & Directly grounded & Nominal speed & $1200 \mathrm{rpm}$ \\
Power factor range & 0.9 lag-0.9 lead & Winding connection & 4 -wire Y \\
\hline
\end{tabular}

Table 3. CTR values for the IEEE 14-bus system.

\begin{tabular}{cccc}
\hline Relay & CTR & Relay & CTR \\
\hline R1 & $200: 1$ & R9 & $900: 1$ \\
R2 & $200: 1$ & $\mathbf{R 1 0}$ & $900: 1$ \\
R3 & $500: 1$ & $\mathbf{R 1 1}$ & $1200: 1$ \\
R4 & $500: 1$ & $\mathbf{R 1 2}$ & $1200: 1$ \\
R5 & $500: 1$ & $\mathbf{R 1 3}$ & $1000: 1$ \\
R6 & $500: 1$ & $\mathbf{R 1 4}$ & $1000: 1$ \\
R7 & $1200: 1$ & $\mathbf{R 1 5}$ & $900: 1$ \\
R8 & $1200: 1$ & $\mathbf{R 1 6}$ & $900: 1$ \\
\hline
\end{tabular}

parallel small DGs having a rated capacity of 1 MVA. The technical data of these DGs are given in Table 2 . The CTR values are also shown in Table 3 .

\subsection{Real industrial radial feeder of TOSEE}

Besides the IEEE 14-bus system which is a meshed network, a real industrial radial feeder named TOSEE is also considered, as shown in Figure 4. This network is one of the outgoing feeders of $63 \mathrm{kV} / 20 \mathrm{kV}$ Koushkan substation located in Zanjan City, Iran. The total load of this system is $17.25 \mathrm{MVA}$ as $15.35 \mathrm{MW}$ and 7.41 MVAr, respectively. This radial feeder is connected to the sub-transmission network via two 40 MVA transformers. It is assumed that the network has three DGs with the capacity of 2 MVA. The CTR values for all the employed DOCRs are 1000:5.

\subsection{Simulation results}

This part presents the simulation results including optimal setting values of DOCRs based on multi- 


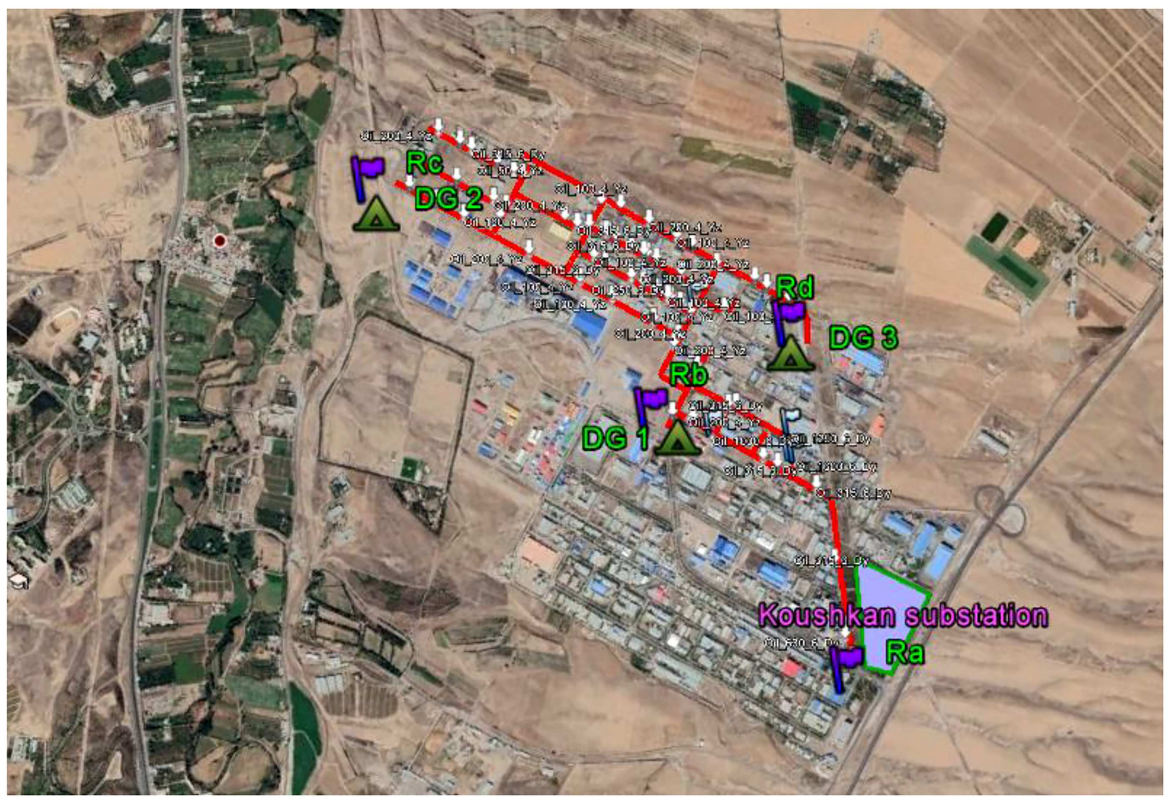

Figure 4. TOSEE industrial radial network with DG units.

Table 4. Cascading outage models.

\begin{tabular}{cc}
\hline Model & Cascading outages \\
\hline 1 & Islanding mode \\
2 & (Substation + DG + line) outages \\
3 & $(2$-case DG) outages \\
4 & $(2$-case line) outages \\
5 & (Substation + DG) outages \\
6 & (Substation + line) outages \\
7 & $($ DG + line $)$ outages \\
\hline
\end{tabular}

objective optimization considering all the cascading outage models (given in Table 4) for the IEEE 14-bus system and TOSEE feeder. The formulation problem investigates all the topologies resulting from every model. For example, in case of DG outage, the relay must support all the currents flowing in the network which resulted from the outage of all three DGs. Also, the current setting value in the first part of the problem has been obtained in terms of maximum current of all the variable topologies. In other words, in case of the outage of three DGs, the one leading to greater current flow on the line has been considered for setting the relay. Moreover, all models involve different particular constraints that must be taken into consideration in the protection coordination programming. The optimal values of decision variables for all DOCRs are obtained and given in Tables 5 and 6 for the IEEE 14-bus system and TOSEE radial feeder, respectively. Also, the total operation time related to each cascading outage for both case studies is demonstrated in Table 7 .
The results for PCP vary for the two networks due to the configuration of these systems (i.e., one is radial and the other is meshed). As a result, the optimal values obtained for TMS in the IEEE 14bus network are higher than those for TOSEE feeder. Furthermore, the number of constraints for the radial feeder is larger than that for the IEEE system (due to the difference in the dimension of the two networks); however, most of them are linear and solving them is straightforward. It must be noted that when the network topology experience a significant outage, the problem constraints are severely changed and become more nonlinear. This issue has a substantial impact on the results leading to a noticeable reduction in the relay's operation speed. As a result, in models with substation outage, it seems that the fault current is considerably lower than the condition that the network is grid-connected. Consequently, the relay operation time increases dramatically. Therefore, the overall operation times shown in Table 7 which are obtained for models $1,2,5$, and 6 are longer than the values obtained for models 3,4 , and 7 . This issue occurs due to the network SCL such that the fault current passing through DOCRs is inversely proportional to the relay's operation time which, in turn, stems from the relay's characteristic curve. Also, the number of problem constraints in models 4 and 7 is higher than that recognized in models 3 and 5 (due to the higher number of lines), but the problem formulation is more comfortable because of the reasons outlined above (i.e., the linear constraints).

In the islanding mode, the number of non-linear constraints becomes too many which makes the problem formulation extremely more complex. Moreover, 
Table 5. Optimal setting values for the IEEE 14-bus system.

\begin{tabular}{|c|c|c|c|c|c|c|c|c|c|c|c|c|c|c|}
\hline \multirow[t]{2}{*}{ Relays } & \multicolumn{2}{|c|}{ Model 1} & \multicolumn{2}{|c|}{ Model 2} & \multicolumn{2}{|c|}{ Model 3} & \multicolumn{2}{|c|}{ Model 4} & \multicolumn{2}{|c|}{ Model 5} & \multicolumn{2}{|c|}{ Model 6} & \multicolumn{2}{|c|}{ Model 7} \\
\hline & $I p$ & $T M S$ & $I p$ & $T M S$ & $I p$ & $T M S$ & $I p$ & $T M S$ & $I p$ & $T M S$ & $I p$ & $T M S$ & $I p$ & $T M S$ \\
\hline R1 & 1.5 & 0.618 & 1.5 & 0.584 & 2.0 & 0.279 & 2.5 & 0.236 & 1.5 & 0.541 & 2.0 & 0.498 & 2.5 & 0.256 \\
\hline R2 & 0.6 & 0.512 & 1.0 & 0.412 & 1.5 & 0.285 & 2.0 & 0.251 & 1.0 & 0.364 & 1.5 & 0.327 & 1.5 & 0.274 \\
\hline R3 & 2.0 & 0.385 & 2.0 & 0.365 & 1.5 & 0.373 & 2.5 & 0.311 & 2.0 & 0.324 & 2.0 & 0.315 & 2.0 & 0.325 \\
\hline $\mathbf{R} 4$ & 0.8 & 0.411 & 1.0 & 0.412 & 2.0 & 0.359 & 2.0 & 0.241 & 1.5 & 0.402 & 2.0 & 0.358 & 2.0 & 0.327 \\
\hline R5 & 1.5 & 0.395 & 1.5 & 0.324 & 1.0 & 0.361 & 2.5 & 0.215 & 1.5 & 0.411 & 1.0 & 0.385 & 1.0 & 0.314 \\
\hline R6 & 1.0 & 0.514 & 1.5 & 0.459 & 2.0 & 0.325 & 2.5 & 0.236 & 1.5 & 0.398 & 2.0 & 0.345 & 2.0 & 0.287 \\
\hline R7 & 1.5 & 0.612 & 2.0 & 0.541 & 1.5 & 0.367 & 1.5 & 0.175 & 1.0 & 0.511 & 1.0 & 0.417 & 2.0 & 0.316 \\
\hline R8 & 1.0 & 0.495 & 1.0 & 0.413 & 2.0 & 0.341 & 2.0 & 0.169 & 1.0 & 0.416 & 1.5 & 0.375 & 0.8 & 0.319 \\
\hline R9 & 2.5 & 0.356 & 2.5 & 0.324 & 2.5 & 0.252 & 2.5 & 0.156 & 2.5 & 0.295 & 2.5 & 0.256 & 2.5 & 0.224 \\
\hline R10 & 2.0 & 0.398 & 1.5 & 0.369 & 2.5 & 0.302 & 2.5 & 0.205 & 0.8 & 0.324 & 1.5 & 0.298 & 2.5 & 0.293 \\
\hline R11 & 1.0 & 0.664 & 2.0 & 0.551 & 2.0 & 0.321 & 1.5 & 0.213 & 2.0 & 0.517 & 2.0 & 0.419 & 2.0 & 0.267 \\
\hline R12 & 1.0 & 0.496 & 1.5 & 0.516 & 2.5 & 0.413 & 2.0 & 0.298 & 1.5 & 0.547 & 2.5 & 0.568 & 2.5 & 0.415 \\
\hline R13 & 1.0 & 0.811 & 2.0 & 0.801 & 1.5 & 0.416 & 2.0 & 0.319 & 1.0 & 0.753 & 1.5 & 0.712 & 1.5 & 0.411 \\
\hline R14 & 1.5 & 0.514 & 1.0 & 0.612 & 2.5 & 0.221 & 2.0 & 0.116 & 2.0 & 0.541 & 2.5 & 0.411 & 1.5 & 0.275 \\
\hline R15 & 0.6 & 0.715 & 1.5 & 0.781 & 1.0 & 0.351 & 1.5 & 0.173 & 1.0 & 0.732 & 1.0 & 0.685 & 2.0 & 0.269 \\
\hline R16 & 0.8 & 0.412 & 1.5 & 0.252 & 1.5 & 0.217 & 2.0 & 0.097 & 1.5 & 0.311 & 1.0 & 0.369 & 2.5 & 0.178 \\
\hline
\end{tabular}

Table 6. Optimal setting values for TOSEE industrial radial feeder.

\begin{tabular}{|c|c|c|c|c|c|c|c|c|c|c|c|c|c|c|}
\hline \multirow[t]{2}{*}{ Relays } & \multicolumn{2}{|c|}{ Model 1} & \multicolumn{2}{|c|}{ Model 2} & \multicolumn{2}{|c|}{ Model 3} & \multicolumn{2}{|c|}{ Model 4} & \multicolumn{2}{|c|}{ Model 5} & \multicolumn{2}{|c|}{ Model 6} & \multicolumn{2}{|c|}{ Model 7} \\
\hline & $I p$ & $T M S$ & $I p$ & $T M S$ & $I p$ & $T M S$ & $I p$ & $T M S$ & $I p$ & $T M S$ & $I p$ & $T M S$ & $I p$ & $T M S$ \\
\hline $\mathbf{R a}$ & 2.0 & 0.253 & 1.5 & 0.325 & 2.0 & 0.197 & 2.5 & 0.143 & 1.5 & 0.312 & 2.0 & 0.297 & 2.5 & 0.167 \\
\hline $\mathbf{R b}$ & 1.0 & 0.221 & 1.0 & 0.312 & 1.5 & 0.156 & 2.0 & 0.102 & 1.5 & 0.261 & 1.5 & 0.243 & 2.0 & 0.125 \\
\hline Rc & 1.0 & 0.088 & 0.5 & 0.126 & 1.0 & 0.081 & 1.5 & 0.077 & 0.5 & 0.087 & 0.8 & 0.084 & 1.5 & 0.078 \\
\hline Rd & 0.8 & 0.152 & 0.5 & 0.198 & 1.5 & 0.124 & 2.5 & 0.109 & 1.0 & 0.178 & 1.0 & 0.174 & 1.5 & 0.111 \\
\hline
\end{tabular}

Table 7. Optimal values for the overall operation time.

\begin{tabular}{|c|c|c|c|c|c|c|c|}
\hline \multirow[t]{2}{*}{ Network } & \multicolumn{7}{|c|}{ Overall operation time } \\
\hline & Model 1 & Model 2 & Model 3 & Model 4 & Model 5 & Model 6 & Model 7 \\
\hline TOSEE feeder & 5.554 & 8.621 & 3.259 & 1.518 & 7.561 & 6.924 & 2.332 \\
\hline IEEE 14-BUS & 41.567 & 28.684 & 16.432 & 10.695 & 26.985 & 23.421 & 14.845 \\
\hline
\end{tabular}

in the islanding mode, there is a significant reduction in SCL such that if not considered, it may lead to instability of the power system.

In TOSEE feeder, as there is only one feeding substation, not only model 1 but also models 2,5 , and 6 lead to the islanding mode. As a result, the overall operation time of these models is longer than that of model 1 (due to 2 or 3 case outages).

Since the fault currents for models 2 and 5 , on the one side, and 3 and 7 , on the other side, are very close to each other, the DOCRs' operation times experience only a trivial difference. Furthermore, model 4 has the fastest reaction time for DOCRs to the fault current, because there is no loss of power source (DGs or substation) in this model.

\subsection{Investigation of the violated coordination}

The purpose of this section is to specify the number of miscoordination occurring as a consequence of solving the PCP. The coordination constraints for DOCRs are considered to be violated when the backup relays operate with a time interval less than 0.3 seconds relative to the main relay (by taking CTI into account). Table 8 shows the number of violated coordinations in each investigated model for the IEEE 14-bus network and TOSEE feeder. It should be noted in both case studies that when DOCRs are set based on models 1 and 2, it supports all topologies in Table 4. Consequently, in the case of such events, the network loses a considerable generation, and if this issue is not taken into account, it leads to an increase in the number of mis-coordinations 
Table 8. Number of violated coordinations.

\begin{tabular}{ccccccccc}
\hline Network & \multicolumn{7}{c}{ Number of mis-coordinations } \\
\cline { 1 - 10 } TOSEE feeder & & Model 1 & Model 2 & Model 3 & Model 4 & Model 5 & Model 6 & Model 7 \\
\cline { 2 - 9 } IEEE 14-BUS & 2 & 3 & 2 & 1 & 2 & 2 & 1 \\
\hline
\end{tabular}

which ends in cascading outages. However, models 5 and 6 applied to the IEEE 14-bus system have 5 and 4 violations, respectively. Similarly, models 5 and 6 applied to TOSEE feeder both have 2 violations. By simplifying the variations of the network topology in models 7 and 4 , there will be the least number of miscoordinations in both networks because the SCL varies gradually.

It is also observed that the number of violated coordinations for the IEEE 14-bus network is larger than that for TOSEE feeder because a larger number of DOCRs are employed in the meshed system and there are higher SCL variations. Finally, if the PCP is set based on the severe outages, it results in an appropriate setting for all the topologies but with a longer operation time.

\subsection{Single-objective optimization}

\subsubsection{Single-objective optimization with optimized} OF coefficients

In this part, for a detailed investigation into the problem, other aspects like single-objective optimization and optimization of objective function coefficients are regarded. To this end, the objective function in [26] is considered as Eq. (12):

$$
\begin{aligned}
O F= & \sum_{d \in D}\left(\left(\alpha \times \sum_{i \in I}\left(t_{i}\right)^{2}\right)+\left(\gamma_{1} \sum_{k \in K} e^{\gamma_{2}\left|\Delta t_{k, d}\right|}\right)\right. \\
& \left.+\left(\beta \sum_{k \in K} B C_{k, d}\right)\right),
\end{aligned}
$$

where $i$ and $k$ indicate all relays and all the main and backup pair relays, respectively. Moreover, $I$ and $K$ represent the sets of all relays and main and backup pair relays, respectively. Further, $d$ is the index of fault points, and $D$ represents the set of fault points. In addition, $B C$ is a binary coefficient that is 1 when the coordination constraint is violated and is 0 otherwise.

The relay operation curve is in line with the IEC standard inverse-time with the same coefficients. Also, the entire coordination procedure is done based on the near end of the fault point to all the main relays. However, to evaluate the problem more, the coefficients of objective function (weighting factors of ) will be optimized by the GA.

According to Table 9, it is observed that the sum of operation times of relays is $11.778 \mathrm{~s}$ which shows a relative increase compared to multi-objective optimization. However, the number of miscoordinations has
Table 9. Obtained results based on the single-objective optimization and coefficient determination.

\begin{tabular}{ccc}
\hline RELAYS & $\boldsymbol{I P}$ & $\boldsymbol{T} \boldsymbol{M} \boldsymbol{S}$ \\
\hline $\mathrm{R} 1$ & 2.5 & 0.234 \\
$\mathrm{R} 2$ & 2 & 0.279 \\
$\mathrm{R} 3$ & 2.5 & 0.328 \\
$\mathrm{R} 4$ & 2 & 0.269 \\
$\mathrm{R} 5$ & 2 & 0.211 \\
$\mathrm{R} 6$ & 1.5 & 0.231 \\
$\mathrm{R} 7$ & 1.5 & 0.165 \\
$\mathrm{R} 8$ & 2 & 0.168 \\
$\mathrm{R} 9$ & 2.5 & 0.216 \\
$\mathrm{R} 10$ & 2.5 & 0.221 \\
$\mathrm{R} 11$ & 1 & 0.216 \\
$\mathrm{R} 12$ & 2 & 0.321 \\
$\mathrm{R} 13$ & 2 & 0.315 \\
$\mathrm{R} 14$ & 2.5 & 0.115 \\
$\mathrm{R} 15$ & 2 & 0.189 \\
$\mathrm{R} 16$ & 2 & 0.116 \\
\hline \multicolumn{2}{c}{ Sum(t) $=11.778$ seconds } \\
Number of mis-coordinations $=1$ \\
$\alpha=10.013 . \gamma_{1}=112.567$, \\
\multicolumn{5}{c}{$\gamma_{2}=108.421$} \\
\hline \multicolumn{3}{c}{}
\end{tabular}

remained to be one case. This shows the capability of the above objective function and its new idea in finding the weighting coefficients using the GA. It should be noticed that by increasing the miscoordination, the value of $\beta$ accordingly increases which reduces the number of violated constraints. The values of the objective function coefficients have been obtained as $\gamma_{1}=112.567, \gamma_{2}=108.421$, and $\alpha=10.013$ by the GA. The obtained results for model 4 of the IEEE 14bus system are as follows.

\subsubsection{Convergence of the proposed method in comparison with single $O F s$}

The convergence trend of the presented method for both case studies is investigated based on MOOM and the single- $O F$. In the single-objective function, all the introduced OFs are considered according to Eq. (13), so that the related constraints are not violated in the absence of these OFs and only the effect of multiobjective optimization and efficiency of the proposed 


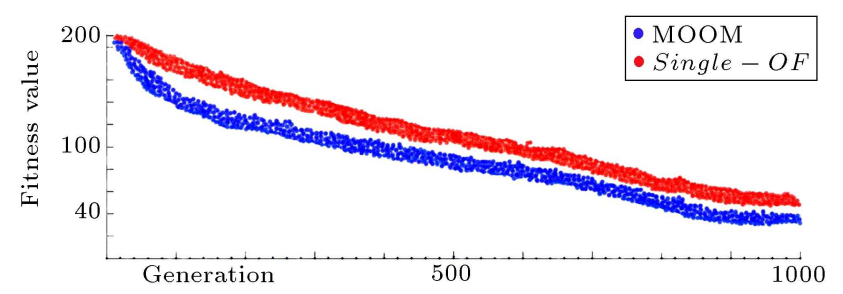

Figure 5. Convergence trend of the proposed method for the islanding mode (model 1) based on MOOM and single-OF in the IEEE 14-bus network.

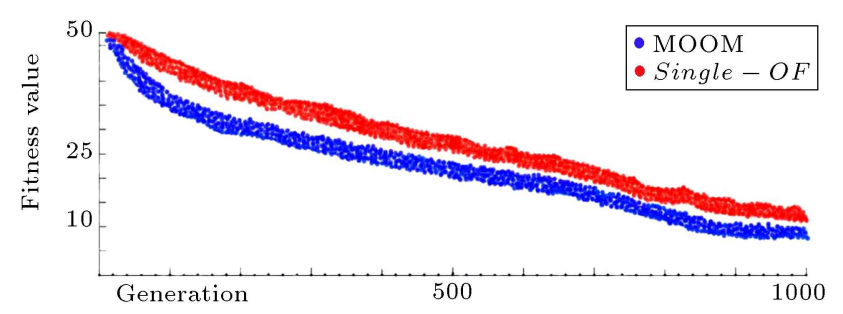

Figure 6. Convergence trend of the proposed method for the islanding mode (model 2) based on MOOM and single-OF in TOSEE radial feeder.

method are observed. Given that the islanding mode:

$$
\text { single }-O F=O F_{A}+O F_{B}+O F_{C}+O F_{D},
$$

is the most hazardous event for both systems; model 1 for the IEEE 14-bus network and model 2 for TOSEE feeder have been chosen for comparison.

As shown in Figure 5, when the problem formulation in the IEEE 14-bus system is based on MOOM, the overall operating time declines by $23 \%$ from 51.15 to 41.567 seconds. Also, according to Figure 6, the DOCRs' operation time in TOSEE feeder decreases from 12.281 to 8.621 seconds which shows a $42 \%$ decline. This reduction in operation time is a considerable value when using MOOM. Furthermore, the effect of MOOM on TOSEE feeder is more than that of the IEEE 14-bus system which is due to the radial structure of the network and the fewer number of non-linear constraints.

\section{Conclusion}

The protection-coordination of DOCRs is a complex and non-linear problem. In the current paper, a new method is proposed based on multi-objective optimization with new objective functions by using GA. Furthermore, the cascading outages were taken into account in the problem formulation. The simulation results demonstrated that considering MOOM and its new OFs would satisfy the problem constraints and increase the operation speed of DOCRs. Provided the multi-objective formulation of PCP, the obtained results for the decision variables (i.e., $T M S$ and $I p$ ) seem to be the best possible values for all of the relays.
The proposed method was applied to the IEEE 14-bus system and a real industrial radial feeder by taking the cascading outages into account. When DOCRs were set having severe outages in mind, they would become suitable for all the single or simultaneous contingencies that may occur. This can help maintain the network security.

\section{Acknowledgment}

The authors acknowledge the support of Hamed Zandi who provided help during this research.

\section{References}

1. Saha, D., Datta, A., Kumar, B., et al. "A comparative study on the computation of directional overcurrent relay coordination in power systems using PSO and TLBO based optimization", Eng. Comput., 33(2), pp. 603-621 (2016).

2. Kheshti, M. and Kang, X. "Optimal overcurrent relay coordination in distribution network based on lightning flash algorithm", Eng. Comput., 35(3), pp. 11401160 (2018).

3. Zeineldin, H., Sharaf, H., Ibrahim, D., et al. "Optimal protection coordination for meshed distribution systems with dg using dual setting directional overcurrent relays", IEEE Trans. Smart Grid, 6(1), pp. 115-123 (2015).

4. Urdaneta, A.J., Nadira, R., and Perez, L.G. "Optimal coordination of directional overcurrent relays in interconnected power systems", IEEE Trans. Power Del., 3(3), pp. 903-911 (1988).

5. Chattopadhyay, B., Sachdev, M.S., and Sidhu, T.S. "An on-line relay coordination algorithm for adaptive protection using linear programming technique", IEEE Trans. Power Del., 11(1), pp. 165-173 (1996).

6. Salazar, C. and Enriquez, A. "Coordination of overcurrent relays using genetic algorithms and unconventional curves", IEEE Latin America Trans., 12(8), pp. 1449-1455 (2014).

7. Najy, W.K.A., Zeineldin, H.H., and Woon, W.L. "Optimal protection coordination for microgrids with grid-connected and islanded capability", IEEE Trans. Ind. Electron., 60(4), pp. 1668-1677 (2013).

8. Marcolino, M., Leite, J., and Mantovani, J. "Optimal coordination of overcurrent directional and distance relays in meshed networks using genetic algorithm", IEEE Latin America Trans., 13(9), pp. 2975-2982 (2015).

9. Shih, M., Salazar, C., and Enríquez, A. "Adaptive directional overcurrent relay coordination using ant colony optimisation", IET Gen, Trans \& Dist., 9(14), pp. 2040-2049 (2015).

10. Zeineldin, H., El-Saadany, E., and Salama, M. "Optimal coordination of overcurrent relays using a modified particle swarm optimization", Electr. Power Syst. Res., 76(11), pp. 988-995 (2006). 
11. Yazdaninejadi, A., Nazarpour, D., and Talavat, V. "Coordination of mixed distance and directional overcurrent relays: mis-coordination elimination by utilizing dual characteristics for DOCRs", Int. Trans. Elect. Energ. Syst., 29(3), p.e2762 (2018).

12. Niranjan, P., Choudhary, N.K., and Singh, R.K. "Performance analysis of different optimization techniques on protection coordination of overcurrent relay in microgrid", Int. Conf. Elect. Electron. Comp. Eng. (UPCON), pp. 1-6 (2019).

13. Saleh, K.A., Zeineldin, H.H., and El-Saadany, E.F. "Optimal protection coordination for microgrid considering n-1 contingency", IEEE Trans. Ind. Informat., 13(5), pp. 2271-2278 (2017).

14. Urdaneta, A.J., Perez, L.G., and Restrepo, H. "Optimal coordination of directional overcurrent relays considering dynamic changes in the network topology", IEEE Trans. Power Del., 12(4), pp. 1458-1464 (1997).

15. Noghabi, A.S., Mashhadi, H.R., and Sadeh, J. "Optimal coordination of directional overcurrent relays considering different network topologies using interval linear programming", IEEE Trans. Power Del., 25(3), pp. 1348-1354 (2010).

16. Madaminov, S. "Fast method for finding N-2 contingencies in power grid", M.Sc Thesis, Skolkovo Institute of Science and Technology (2015).

17. Union for Coordination of Transmission of Electricity (UCTE), Final Report System Disturbance on 4 November 2006, UCTE, Brussel, Belgium, www.ucte.org. (2006).

18. Central Electricity Regulatory Commission, Report on the Grid Disturbance on 30th July and 31st Jul. 2012, www.cercind.gov.in (2012).

19. EATON. Power Outage Annual Report 2013 (2013).

20. Liu, Y., Li, Y., Sheng, M., et al., "Reliability prediction method and application in distribution system based on genetic algorithm-back-propagation neural network", IET Gen, Trans \& Dist, 13(7), pp. 984-988 (2019).

21. Yazdaninejadi, A., Nazarpour, D., and Talavat, V. "Optimal coordination of dual-setting directional overcurrent relays in multi-source meshed active distribution networks considering transient stability", IET Gen, Trans \& Dist, 13(2), pp. 157-170 (2019).

22. Durand, D. and Pieniazek, D. "Overcurrent protection \& coordination for industrial applications", 2010 Industry Applications Society Annual Meeting (2010).
23. Celli, G., Pilo, F., Pisano, G., et al. "Distribution energy storage investment prioritization with a real coded multi-objective genetic algorithm", Electr. Power Syst. Res., 163, pp. 154-163 (2018).

24. Yazdanpanahi, H., Xu, W., and Li, Y. "A novel fault current control scheme to reduce synchronous dg's impact on protection coordination", IEEE Trans. Power Del., 29(2), pp. 542-551 (2014).

25. University of Washington, Seattle "Power Systems Test Case Archive", Available at labs.ece.uw. edu/pstca (1993).

26. Yazdaninejadi, A., Naderi, M.S., Gharehpetian, G.B., et al. "Protection coordination of directional overcurrent relays: new time current characteristic and objective function", IET Gen, Trans \& Dist, 12(1), pp. 190-199 (2018).

\section{Biographies}

Hossein Karimkhan Zand was born in 1993 in Zanjan, Iran. He received his BSc and MSc degrees in Power Engineering from the University of Zanjan in 2016 and 2019, respectively. He is currently a GIS expert with the Zanjan Electricity Distribution Company (ZEDC). His research interests include power system protection, renewable energies, and cyber-attacks.

Kazem Mazlumi was born in Tehran, Iran in 1976. $\mathrm{He}$ received the $\mathrm{BSc}$ degree in Electrical Engineering from the Amirkabir University of Technology, Tehran in 2000, the MSc degree from the Sharif University of Technology, Tehran in 2003, and the PhD degree from the Amirkabir University of Technology in 2009. He is currently an Associate Professor at the University of Zanjan, Zanjan, Iran. His research interests include power system protection and smart power grids.

Amir Bagheri was born in 1984, Zanjan, Iran. He received his MSc and $\mathrm{PhD}$ degrees in Power Engineering from the University of Zanjan and the University of Tehran in 2010 and 2015, respectively. He is currently an Assistant Professor at the University of Zanjan. His research area covers power system planning and operation, renewable energies, and optimization algorithms. 